\title{
Paper-Based ELISA
}

\section{Citation}

Cheng, Chao-Min, Andres[W. Martinez, Jinlong Gong, Charles RR. Mace, Scottपा. Phillips, Emanuel Carrilho, Katherine[A. Mirica, and George[M. Whitesides. 2010. "Paper-Based ELISA." Angewandte Chemie 122 (28) (May 28): 4881-4884. doi:10.1002/ange.201001005.

\section{Published Version}

doi:10.1002/ange. 201001005

\section{Permanent link}

http://nrs.harvard.edu/urn-3:HUL.InstRepos:33490478

\section{Terms of Use}

This article was downloaded from Harvard University's DASH repository, and is made available under the terms and conditions applicable to Open Access Policy Articles, as set forth at http:// nrs.harvard.edu/urn-3:HUL.InstRepos:dash.current.terms-of-use\#OAP

\section{Share Your Story}

The Harvard community has made this article openly available.

Please share how this access benefits you. Submit a story.

Accessibility 


\title{
Paper-Based ELISA
}

Chao-Min Cheng, ${ }^{1 \#}$ Andres W. Martinez, ${ }^{1 \#}$ Jinlong Gong, ${ }^{1}$ Charles R. Mace, ${ }^{1}$ Scott T. Phillips, ${ }^{1}$ Emanuel Carrilho, ${ }^{1,2}$ Katherine A. Mirica, ${ }^{1}$ and George M. Whitesides ${ }^{1, *}$

${ }^{1}$ Department of Chemistry \& Chemical Biology, Harvard University, Cambridge, MA 02138

\author{
${ }^{2}$ Instituto de Química de São Carlos, Universidade de São Paulo \\ 13566-590 São Carlos - SP, Brazil
}

*Corresponding author E-mail: gwhitesides@gmwgroup.harvard.edu

"These authors contributed equally to this work. 
This paper describes enzyme-linked immunosorbent assays (ELISA) performed in a 96-microzone plate made out of paper (paper-based ELISA, or P-ELISA). ELISA is widely used in biochemical analyses, including immunoassays, food industry assays for food allergens, and toxicological assays. These assays are typically carried out in microtiter plates or small vials. ${ }^{1,2}$ ELISA combines the specificity of antibodies and the high-turnover catalysis by enzymes, to provide specificity and sensitivity. ${ }^{1,2}$ We have recently described a 96-microzone paper plate—fabricated by patterning hydrophobic polymer in hydrophilic paper - as a platform for biochemical analysis. ${ }^{3,4}$ Although microfluidic paper-based analytical devices ( $\mu$ PADs) were designed primarily to provide analytical capability at low cost in developing countries, ${ }^{5-7}$ we expect that they will also be useful in applications such as point-of-care clinical analysis, military field operations, and others where high throughput, low volumes of sample, low cost, and robustness are important. ${ }^{6,7}$ These devices have so far been prototyped using analyses of simple metabolites: glucose, protein, and certain enzymes. ${ }^{8-10}$ P-ELISA combines the sensitivity and specificity of ELISA with the convenience, low cost and ease-of-use of paper-based platforms.

Porous membranes, including nitrocellulose and filter paper, have been used for decades in dot-immunobinding assays (DIA). ${ }^{8-13}$ Though DIAs are the simplest form of immunoassays on paper, they typically require one piece of nitrocellulose for each assay, the pieces of nitrocellulose have to be processed individually in Petri dishes, and the assays take several hours to complete. ${ }^{9}$ Quantitative DIAs have been reported, ${ }^{14}$ but DIAs are typically qualitative, and provide only "yes/no" results. ${ }^{15}$ Traditional ELISA, usually performed in 96-well plates (fabricated by injection molding in plastic), is quantitative 
and well-suited for high-throughput assays, but each assay requires large volumes $(\sim 20$ $200 \mu \mathrm{L}$ ) of analyte and reagents, the incubation and blocking steps are long ( $\geq 1 \mathrm{~h}$ per step, because the reagents must diffuse to the surface of the wells), and the results are quantified using a plate reader, typically an $\sim \$ 20,000$ instrument. ${ }^{9,16}$

Paper microzone plates for ELISA can have the same layout as plastic 96-well plates, but each test zone requires only $\sim 3 \mu \mathrm{L}$ of sample, and the results can be measured using a desktop scanner, typically a $\sim \$ 100$ instrument. In addition, an entire P-ELISA can be completed in less than one hour. The ease of fabrication of paper microzone plates also opens opportunities for a wide range of non-standard formats, and customized connections to carry reagents between zones. To evaluate the feasibility of P-ELISA, and the potential advantages and disadvantages of P-ELISA and 96-well-plate-based ELISA, we developed a three-step procedure that i) immobilizes targeted antigens and then incubates them with their primary antibodies on a 96-microzone paper plate; ii) limits non-specific binding of proteins in the test zones (so-called 'blocking'); and iii) washes unbound proteins out of the test zone. Once these protocols were established, we demonstrated an indirect P-ELISA using rabbit IgG as a model analyte. We also established that P-ELISA can be used to detect and quantify HIV-1 envelope (gp41) antigen in human serum through immobilizing the HIV-1 envelope antigen (gp41) on paper and then using anti-HIV-1 antibody conjugated to alkaline phosphatase (ALP) for producing a colorimetric readout.

We first designed a 96-microzone paper plate with an array $(12 \times 8)$ of circular test zones for running multiple P-ELISAs in parallel, and fabricated it by photolithography using Whatman No. 1 Chromatography paper and SU-8 as a photoresist 
(Figure 1A) ${ }^{17}$ A range of methods of fabricating $\mu$ PADs have been developed ${ }^{7,17}$, and in different circumstances, different methods may be preferred. The array was designed to have the same layout and dimensions as a standard plastic 96-well plate, so that it would be compatible with existing microanalytical infrastructure (eight- or twelve-channel pipettes and plate readers). Each test zone was $5 \mathrm{~mm}$ in diameter and required $3 \mu \mathrm{L}$ of solution to fill (e.g., to wet completely with fluid); this design was a good compromise between convenience and conservation of reagents, as it reduced the amount of reagents and sample required for the assay, but ensured accurate distribution of fluids when using a manual pipette.

We also examined smaller test zones, the smallest test zone required $0.5 \mu \mathrm{L}$ of solution to fill. This volume is similar to that required in a 384-well plate format. Even though these smaller test zones showed results comparable to the larger test zones, we chose to work with the larger size because: (i) the 96-well plate format is so widely used and so familiar to those with potential interests in this platform, and (ii) the requirement of robotic sample application in arrays of smaller zones would limit its application to technically sophisticated laboratories.

The top and bottom faces of the test zones in paper-microzone plates are open to atmosphere. ${ }^{4}$ The advantage of this configuration is that the zones can be washed by adding a washing buffer to the top of the zone while pressing the bottom of the zone against a piece of blotting paper. The washing buffer wicks through the test zone and into the blotting paper carrying unbound reagents with it. The disadvantage of this configuration of the paper-microzone plate is that when reagent solutions are added to the test zones, the paper plate must be suspended in air to prevent the reagent solutions from 
wicking through the test zones. Empty pipette-tip boxes provided a simple platform for supporting 96-microzone paper plates such that the test zones are completely suspended and isolated.

We chose a colorimetric readout so that the results of the P-ELISA could be interpreted without the need for a specialized detector (unlike fluorescence readout). Colorimetric assays can be read visually, and/or quantified using a scanner or camera phone. ${ }^{6}$ These assays are thus well-suited for use in settings lacking expensive microtiter plate readers or fluorescence scanners. There are several examples of enzyme/substrate pairs that have been used in conventional ELISA to produce colored products; ${ }^{18,19}$ we chose alkaline phosphatase as the enzyme, and BCIP/NBT (5-bromo-4-chloro-3-indolyl phosphate and nitro blue tetrazolium $)^{19}$ as the substrate, because they produce a color change from light yellow to dark purple, and thus have excellent contrast with the white background provided by paper. This system has been extensively characterized and benefits from the commercial availability of a range of ALP-conjugated antibodies. ${ }^{20}$

In its simplest form, indirect P-ELISA comprises five steps: (i) immobilizing the antigen on paper, (ii) blocking the paper to prevent non-specific adsorption of proteins, (iii) labeling the immobilized antigens with an enzyme-conjugated detection antibody, (iv) washing away unbound antibody, and (v) adding a solution of a substrate for the enzyme (Figure 1B). To immobilize the antigen on patterned paper, we added $3 \mu \mathrm{L}$ of a solution of the rabbit IgG in phosphate buffered saline (PBS) to the test zone, and allowed it to dry for 10 minutes under ambient conditions. The test zones were then blocked by adding $3 \mu \mathrm{L}$ of a blocking buffer [0.05\% (w/v) Tween-20 and 1\% (w/v) bovine serum albumin (BSA) in PBS] to each zone, and allowing it to dry for 10 minutes 
under ambient conditions. The enzyme-linked antibody in an incubation buffer $(3 \mu \mathrm{L}$, $0.05 \%$ Tween-20 in PBS) was added to each zone and allowed to incubate for one minute. Each test zone was washed twice by adding $10 \mu \mathrm{L}$ of PBS to the test zone, and then bringing the bottom of the paper-microzone plate in contact with a piece of blotting paper to absorb the excess wash buffer from the test zone. Finally, $3 \mu \mathrm{L}$ of a solution of the colorimetric substrate for ALP (2.7-mM 5-bromo-4-chloro-3-indolyl phosphate disodium salt, 1.8-mM nitrotetrazolium blue chloride, 5-mM $\mathrm{MgCl}_{2}, 100-\mathrm{mM} \mathrm{NaCl}$, $0.05 \%$ Tween in $100-\mathrm{mM}$ Tris buffer, $\mathrm{pH} 9.5)^{21}$ was added to the test zone immediately after the washing step, and the color-producing enzymatic reaction was allowed to proceed for 30 minutes under ambient conditions. The test zone was scanned, and the intensity of the color was measured using ImageJ. ${ }^{22,23}$

Rabbit IgG adsorbed on the surface of the cellulose fibers of the paper-based test zones when a solution of the protein was allowed to dry in the test zone under ambient conditions for 10 minutes. We tested the ability to adsorb antigens and antibodies on paper using solutions of rabbit IgG labeled with tetramethyl rhodamine iso-thiocyanate (TRITC). TRITC-IgG provided a useful test protein because it could be quantified directly on the paper using a fluorescence scanner without the need for enzymatic catalysis for generating colorimetric readout. The fluorescence signals of test zones that had been spotted with solutions of TRITC-labeled rabbit IgG, dried, and then washed with PBS were indistinguishable from the fluorescence signals of test zones that had been spotted with TRITC-IgG and not washed. The similarity of these two results indicated that the antibodies were adsorbed to the paper (Figure 1C), and did not elute with the washing buffer. Spotting $1 \mu \mathrm{L}$ 1\% BSA blocking buffer on the paper test zones and 
allowing the buffer to dry blocked non-specific binding of subsequently added proteins to paper (Figure 1D). Solutions of TRITC- rabbit IgG were added to test zones that had been blocked, or not blocked, with the blocking buffer, allowed to incubate for a minute, and then washed. The blocked test zones had fluorescent signals that were equivalent to the background signal; this observation indicated that the blocking step prevented nonspecific binding of antibodies to paper. We then tested nine different combinations of washes for removing unbound proteins from paper (Figure 1E). A solution of TRITCrabbit $\operatorname{IgG}(50 \mathrm{ng} / \mu \mathrm{L})$ was incubated on blocked test zones for one minute and then washed. Though several different combinations of washes provided complete removal of the labeled antibody, we chose to wash the test zones two times with $10-\mu \mathrm{L}$ volumes of PBS, as this procedure effectively removed traces of non-specifically bound protein while minimizing the time these steps contributed to the assay. 
Figure 1. Prototype demonstration of an indirect P-ELISA. A) A paper 96-microzone plate for P-ELISA designed with the equivalent dimensions of a 96-well microtiter plate. Each circular test zones of hydrophilic paper is bounded by hydrophobic photoresist. B) Schematic diagram of a general paper-based indirect ELISA utilizing antigen/antibody recognition. TRITC-labeled rabbit IgG was used to develop a protocol for immobilizing proteins on paper for P-ELISA. C) Comparison of the fluorescence signal from test zones with immobilized TRITC-labeled rabbit IgG that were either washed or not washed (control). The height of the bars for all the results shown in (C), (D) and (E) represent the average normalized fluorescence intensity of eight independent measurements, and the error bars represent one standard deviation from the average. All the results shown in (C), (D) and (E) were normalized to the average fluorescence intensity of $50 \mathrm{ng}$ of TRITCrabbit IgG immobilized on paper. D) Comparison of the fluorescence intensity from test zones that were either blocked or not blocked (control), then incubated with solutions of TRITC-rabbit IgG for one minute and washed. E) Comparison of the fluorescence intensity from test zones that were blocked, incubated with 50-ng/ $\mu \mathrm{L}$ TRITC-rabbit IgG for one minute and then washed with PBS using different protocols. 
Figure 1.
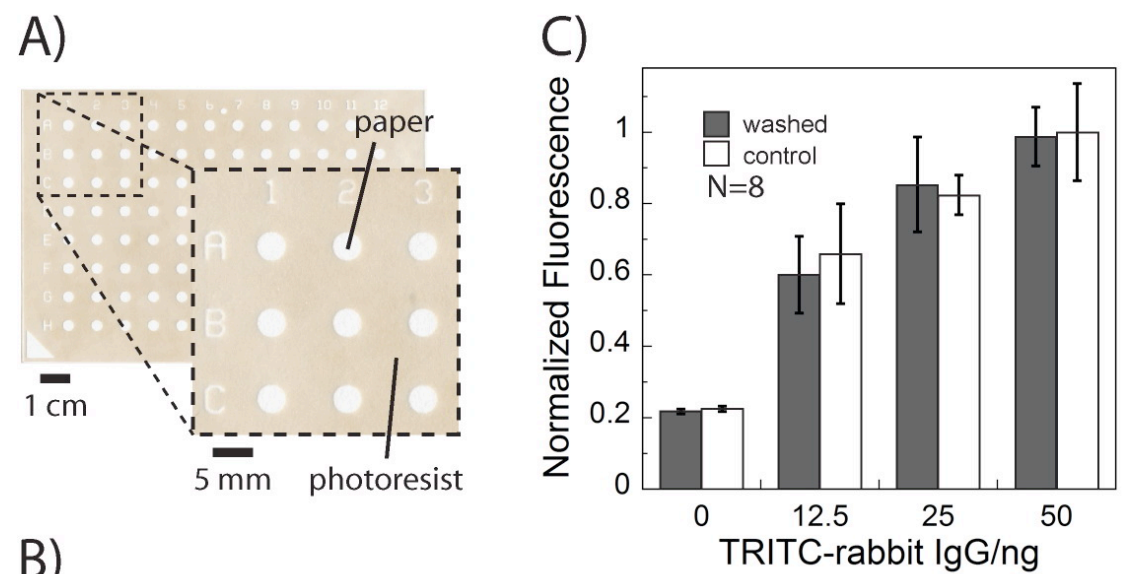

B)
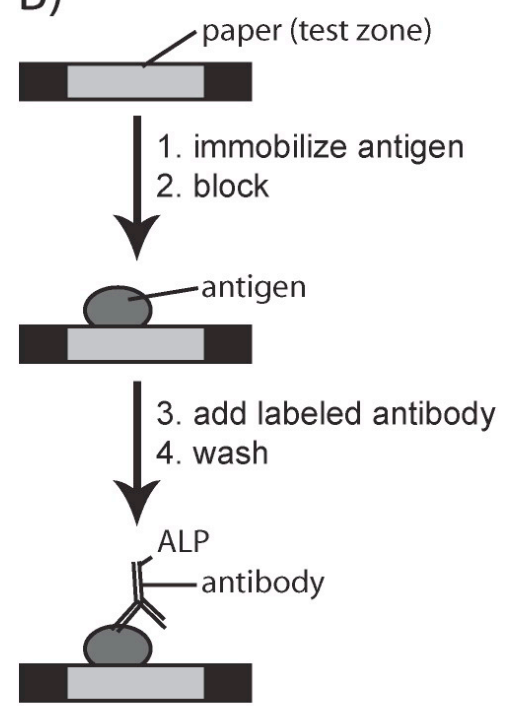

D)

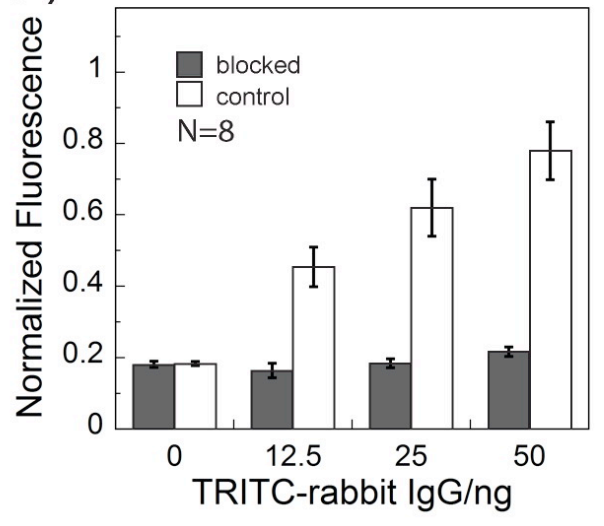

E)

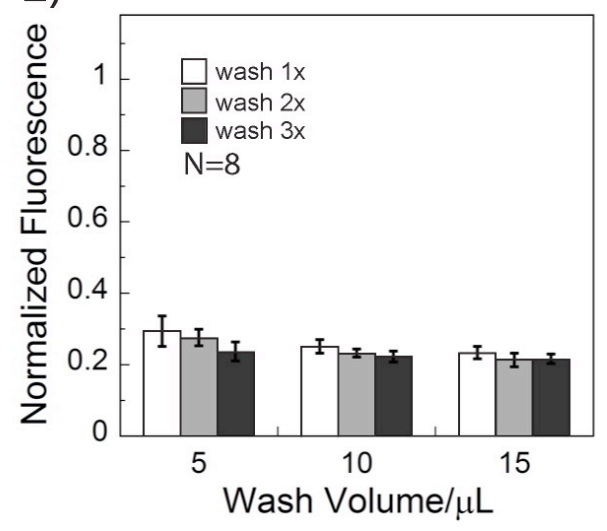


We carried out P-ELISA for the detection of rabbit IgG in 10-fold dilutions (67 $\mathrm{mM}$ to $670 \mathrm{nM}$ per zone, corresponding to 67 pmoles to 670 amoles of IgG per zone spotted on the paper) (Figure 2). The intensity of the purple color that developed in each test zone was proportional to the amount of rabbit IgG adsorbed on the paper. We analyzed the data from the dilution series by non-linear regression using the Hill Equation (eq. 1) to generate a sigmoidal curve fit.

$$
\theta=\frac{[L]^{n}}{[L]^{n}+\left[K_{D}\right]^{n}}
$$

In this equation, $\theta$ is the fraction of occupied binding sites, $[L]$ is the ligand concentration (molar), $\left[L_{50}\right]$ is the ligand concentration corresponding to half of the binding sites occupied (molar), and $n$ describes the slope of the curve. The intensity, $I$, of the observed signal, developed colorimetrically by the ALP conjugate, is proportional the number of detected rabbit $\operatorname{IgG}\left(\theta=\frac{I}{I_{\max }}\right)$, such that the fraction of occupied binding sites can be represented by the ratio of the observed intensity to the maximum intensity (eq. 2).

$$
I=\frac{I_{\max }[L]^{n}}{[L]^{n}+\left[K_{D}\right]^{n}}
$$

We determined the limit of detection of rabbit IgG using an indirect P-ELISA to be 54 fmoles/zone, as defined by the concentration that results in a signal that is three times the standard deviation of the control ( $3 \sigma$ rule). This sensitivity is lower than that obtained by ELISA experiments in 96-well plates for the same antigen/antibody pair (4 fmoles/zone, as detected by absorbance). ${ }^{16}$ The difference in the detection limit for P-ELISA may be a consequence of the abbreviated antibody/antigen incubation period (lower loading) or 
non-specific interactions between ALP and the cellulose fibers (high deviations in the background signal).

Paper-based ELISA has three advantages over traditional ELISA that make it an attractive option for routine use (Table 1): (i) An entire paper-based ELISA (from antigen immobilization to final quantitative result) can be completed in less than one hour; traditional ELISA requires at least one hour of incubation just for immobilizing the antigen on the surface of the 96-well plate, (ii) paper-based ELISA requires less than 20 $\mu \mathrm{L}$ of reagents to perform the full assay, and (iii) the results of paper-based ELISA could be quantified with a desktop scanner rather than the more expensive microtiter plate reader.

The small volume requirements of P-ELISA can be attributed to the small volume of the test zone. A paper test zone has a volume of $3.5 \mu \mathrm{L}$ (circular test zone with a diameter of $5 \mathrm{~mm}$ and a height of $0.18 \mathrm{~mm}$ ), but approximately $30 \%$ of this volume is occupied by the paper fibers. The short incubation times for P-ELISA can be attributed to the high surface-to-volume ratio of the paper fibers in the test zone; the antigens, antibodies and reagents have only short distances over which they must diffuse to reach the surface of a paper fiber. Furthermore, as the solutions dry in the test zones, the concentration of each reagent increases, further enhancing the antibody/antigen binding kinetics. One disadvantage of working with test zones that are open to atmosphere is that the rate of evaporation of water from the test zones is dependent on the ambient relative humidity and temperature, and these environmental conditions may affect the results of the P-ELISA. 
Figure 2. Paper-based ELISA for rabbit IgG. A) Schematic of the paper-based indirect ELISA for rabbit IgG. B) Images of the indirect ELISA results from 670 amoles to 67 pmoles of rabbit IgG per zone. C) Calibration plot for the mean intensity of the color produced by the enzymatic reaction of alkaline phosphatase (ALP) in the indirect ELISA assay versus the amount of rabbit IgG adsorbed to each microzone. Each datum is the mean of eight replicates, and the error bars represent the standard deviations of the measurements. We used Image ${ }^{23}$ to analyze the intensity of the color of each test zone after scanning the paper 96-microzone plate. The $\mathrm{R}^{2}$ value of the curve fit to the data using the Hill Equation is 0.992. In addition, we can approximate the curve as linear between the concentrations of $10^{1}-10^{4}$ fmoles/zone. 
Figure 2.

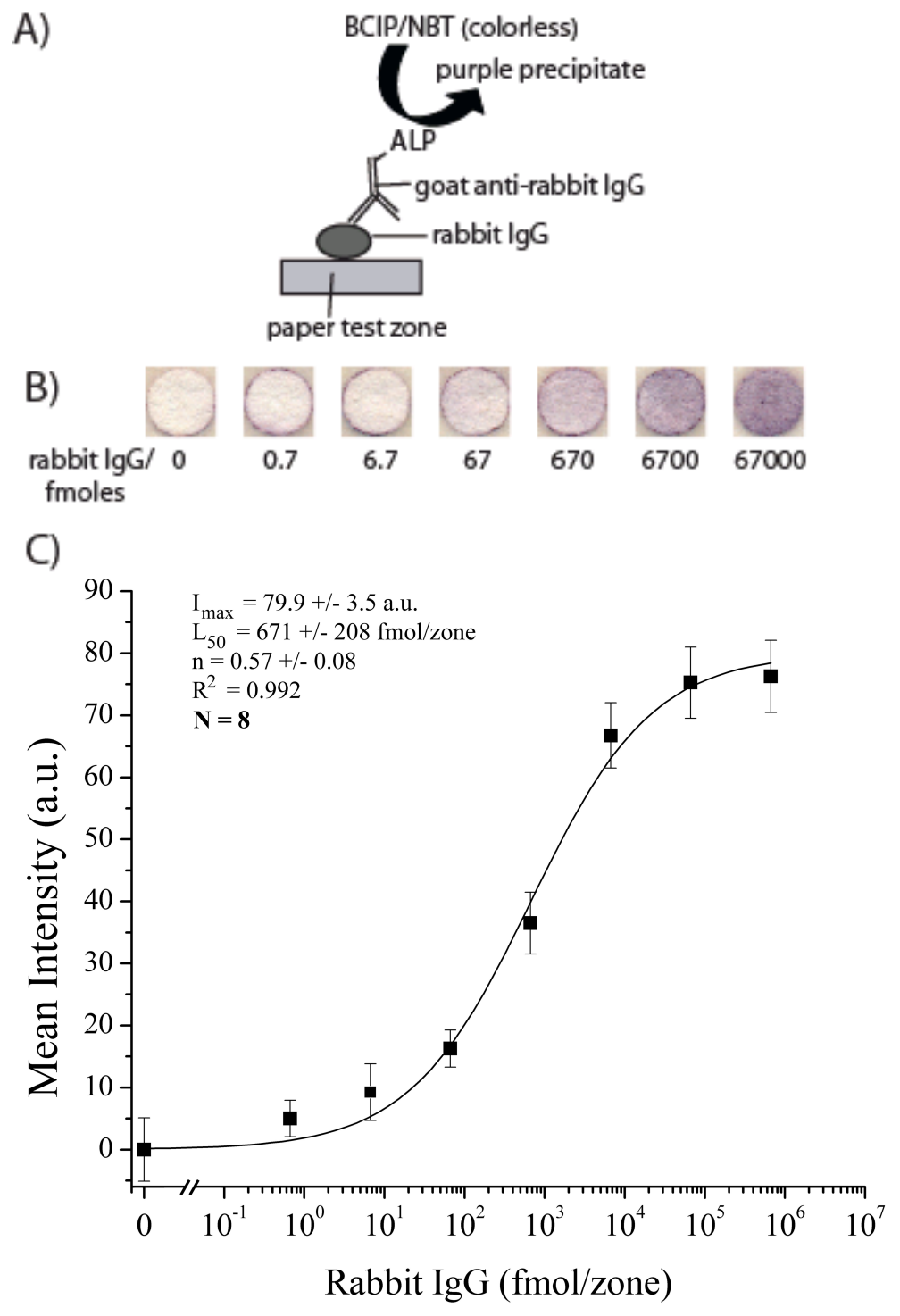


Table 1. Comparison of paper-based ELISA and traditional ELISA. ${ }^{16}$

\begin{tabular}{|c|c|c|c|c|}
\hline \multirow[b]{2}{*}{ Antigen/Antibody } & \multicolumn{2}{|c|}{ Paper-based ELISA } & \multicolumn{2}{|c|}{ Traditional ELISA } \\
\hline & \multicolumn{2}{|c|}{ rabbit IgG / goat IgG } & \multicolumn{2}{|c|}{ rabbit IgG / goat IgG } \\
\hline Enzyme/Substrate & \multirow{2}{*}{\multicolumn{2}{|c|}{$\begin{array}{l}\text { ALP / BCIP/NBT } \\
\text { desktop scanner }(\$ 100)\end{array}$}} & \multicolumn{2}{|c|}{ ALP / nitrophenyl phosp } \\
\hline Detection Device & & & plate reader $(\$$ & $0,000)$ \\
\hline Sensitivity & 54 fmoles/zone & & 4 fmoles/zone & \\
\hline Time and reagents & Volume $(\mu \mathrm{L})$ & Time (min) & $\underline{\text { Volume }(\mu \mathrm{L})}$ & Time $(\min )$ \\
\hline 1) Antigen immobilization & 3 & 10 & 70 & 120 \\
\hline 2) Blocking & 3 & 10 & 100 & 30 \\
\hline 3) Antibody complexing & 3 & 1 & 30 & 60 \\
\hline 4) Signal amplification & 3 & 30 & 100 & 3 \\
\hline Total per Zone & 12 & 51 & 300 & 213 \\
\hline
\end{tabular}


We also demonstrated that P-ELISA could be used to detect HIV-1 envelope (gp41) antigen in human serum. Figure 3A outlines the detection process; this procedure is the same as that we developed to detect rabbit IgG. We examined a number of HIV-1 positive samples with known diluted concentrations, and control samples (human serum without HIV-1 envelope antigen); results indicated that colorimetric signal intensities of both samples decrease upon two sets of 10-fold dilutions (Figures 3B, C). The positive result could be distinguished even for a ten-fold dilution of the human serum; this result suggests that it is practical to use quantitative paper-based ELISA to measure HIV-1 envelope antigen in human serum.

The combination of ELISA and patterned paper provides a new protocol for performing immunoassays. P-ELISA offers three principal advantages over conventional ELISA in plastic 96-well plates: (i) the assay is rapid and can be completed in less than 1 h, (ii) only requires small volumes ( $\sim 1-10 \mu \mathrm{L})$ of samples and reagents, (iii) and utilizes simple equipment $—$ a pipette, a refrigerator for storing the reagents, and a scanner. PELISA thus extends the application of ELISA, especially in small laboratories and in developing countries. The main disadvantage of P-ELISA is that it is less sensitive than traditional ELISA by approximately an order of magnitude ( $54 \mathrm{fmol} / \mathrm{zone}$ vs. 4 fmol/zone). The limit of detection for the P-ELISA approach may be greatly improved by further optimizing the materials and protocols used for the assay in order to suppress the background signal; for example, the type of paper, the blocking buffer, the washing buffer, and the enzyme/substrate pair potentially contribute to producing background intensity. The methods that we demonstrate here for P-ELISA should be broadly adaptable to other immunoassay strategies and labeled bioassays. As an additional 
advantage, the methods developed for P-ELISA lay the foundation for new paper-based

point-of-care immunoassays using microfluidic paper-based analytical devices ( $\mu$ PADs).

\section{ACKNOWLEDGEMEnTS}

This work was funded by the Bill \& Melinda Gates Foundation under award number 51308, by the Micro-Nano Fluidics Fundamentals Focus Center (MF3) at the University of California, Irvine, and by a visiting scholar fellowship from the Fundação de Amparo à Pesquisa do Estado de São Paulo-FAPESP, Brazil (E.C.).

\section{EXPERIMENTAL SECTION}

Details of experimental methods are given in the Supporting Information. 
Figure 3. Paper-based ELISA for HIV-1 envelope (gp41) antigen. A) Schematic diagram of the antibodies and antigens in the assay. HIV-1 envelope antigens came from the serum samples. B) Results of assays for serum from HIV-1 positive patients and control patients. The positive result could be distinguished even for a ten-fold dilution of the serum. C) Graph of the mean intensity of the color developed in the test zones for the assays. The height of the bars represent the average of eight independent measurements, the error bars represent one standard deviation from the average. 
Figure 3.

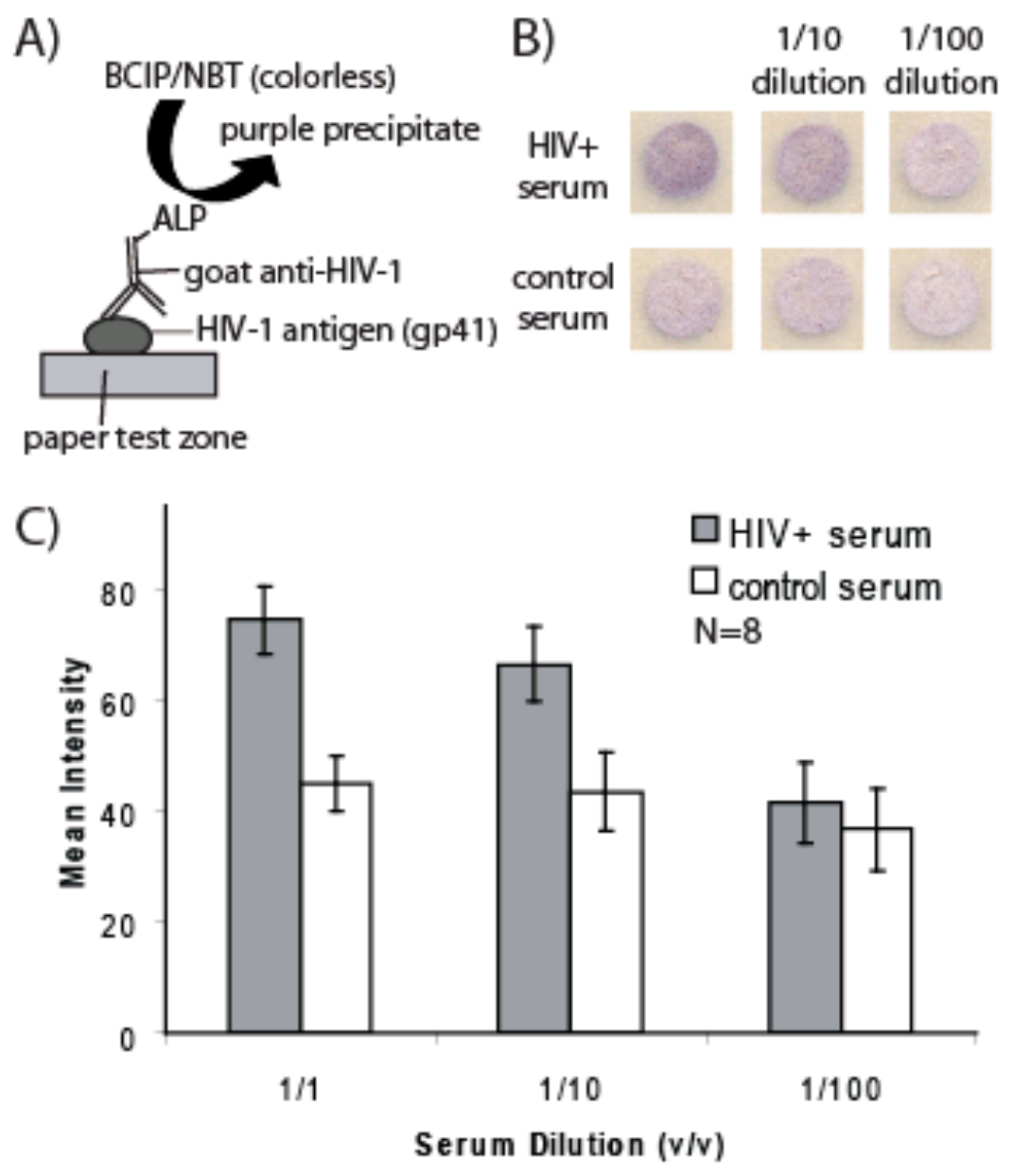




\section{REFERENCES}

(1) R. Edwards, Immunodiagnostics, Oxford University Press, Oxford, UK, 1999.

(2) E. P. Diamandis, Immunoassay, Academic Press, St. Louis, MO, 1996.

(3) A. W. Martinez, S. T. Phillips, M. J. Butte and G. M. Whitesides, Angew. Chem. Int.

Ed. 2007, 46, 1318-1320.

(4) E. Carrilho, S. T. Phillips, S. J. Vella, A. W. Martinez, G. M. Whitesides, Anal. Chem. 2009, 81, 5990-5998.

(5) W. Zhao, A. van der Berg, Lab Chip 2008, 8, 1988-1991.

(6) A. W. Martinez, S. T. Phillips, E. Carrilho, S. W. Thomas, H. Sindi, G. M.

Whitesides, Anal. Chem. 2008, 80, 3699-3707.

(7) A. W. Martinez, S. T. Phillips, G. M. Whitesides, Proc. Natl. Acad. Sci. USA 2008, $105,19606-19611$.

(8) R. Hawkes, Methods Enzymol. 1986, 121, 484-491.

(9) S. Sumi, A. Mathai, V. V. Radhakrishnan, Methods Mol. Biol. 2009, 536, 89-93.

(10) J. Fletcher, Appl. Environ. Microbiol. 1987, 53, 183-184.

(11) C. F. Beyer, J Immunol. Methods 1984, 67, 79-87.

(12) R. L. Heberling, S. S. Kalter, Dev. Biol. Stand. 1986, 64, 199-203.

(13) R. Hawkes, E. Niday, J. Gordon, Anal. Biochem. 1982, 119, 142-147.

(14) R. Jahn, W. Schiebler, P. Greengard, Proc. Natl. Acad. Sci. USA 1984, 81, $1684-$ 1687.

(15) S. S. Kalter, R. L. Heberling, J. D. Barry, I. K. Kuramoto, P. V. Holland, K. Sazama, J Clin. Microbiol. 1992, 30, 993-995. 
(16) S. K. Sia, V. Linder, B. A. Parviz, A. Siegel, G. M. Whitesides, Angew. Chem. Int.

Ed. 2004, 43, 498-502.

(17) A. W. Martinez, S. T. Phillips, B. J. Wiley, M. Gupta, G. M. Whitesides, Lab Chip 2008, $8,2146-2150$.

(18) E. D. Hilborn, W. W. Carmichael, M. Yuan, S. Azevedo, Toxicon 2005, 46, 218-221.

(19) N. van Poperin, D. E. Lopatin, J Clin. Microbiol. 1991, 29, 2554-2558.

(20) J. J. Leary, D. J. Brigati, D. C. Ward, Proc. Natl. Acad. Sci. USA 1983, 80, 40454049.

(21) J. McGadey, Histochemie 1970, 23, 180-184.

(22) M. D. Abramoff, P. J. Magelhals, S. J. Ram, Biophotonics Int. 2004, 11, 36-42.

(23) public software from National Institutes of Health; http://rsbweb.nih.gov/ij/ 
$\underline{\text { Supporting Information }}$

\section{Paper-Based ELISA}

Chao-Min Cheng, ${ }^{1 \#}$ Andres W. Martinez, ${ }^{1 \#}$ Jinlong Gong, ${ }^{1}$ Charles R. Mace, ${ }^{1}$ Scott T. Phillips, ${ }^{1}$ Emanuel Carrilho, ${ }^{1,2}$ Katherine A. Mirica, ${ }^{1}$ and George M. Whitesides ${ }^{1, *}$

${ }^{1}$ Department of Chemistry \& Chemical Biology, Harvard University, Cambridge, MA 02138

${ }^{2}$ Instituto de Química de São Carlos, Universidade de São Paulo 13566-590 São Carlos - SP, Brazil

*Corresponding author E-mail: gwhitesides@gmwgroup.harvard.edu

"These authors contributed equally to this work. 


\section{EXPERIMENTAL Details}

Fabrication of a Paper 96-Microzone Plate. We fabricated the 96-microzone paper plate using photolithography. Paper was impregnated with SU-8 photoresist (MicroChem), baked on a hot plate $\left(10\right.$ minutes, $\left.110^{\circ} \mathrm{C}\right)$, cooled to room temperature and exposed to UV light (13 seconds, IntelliRay 600, UVitron International, Inc.) through a transparency mask. The paper was then baked a second time $\left(5\right.$ minutes, $\left.110^{\circ} \mathrm{C}\right)$, cooled, and the patterns were developed in an acetone bath ( 1 minute) followed by a rinse in acetone and a rinse in 70\% isopropyl alcohol. The paper was blotted between two paper towels, rinsed a second time with $70 \%$ isopropyl alcohol, blotted, and allowed to dry under ambient conditions for at least 1 hour before use.

Immobilizing Proteins on Paper. Solutions of 50-ng/ $\mathrm{L}$, $25-\mathrm{ng} / \mu \mathrm{L}, 12.5-\mathrm{ng} / \mu \mathrm{L}$, and 0 -ng/ $\mu$ LTRITC-labeled rabbit IgG in PBS $(3 \mu \mathrm{L})$ were spotted in paper test zones and allowed to dry under ambient conditions. Half of the test zones were washed with PBS $(2 \times 10 \mu \mathrm{L})$ by adding PBS to the test zone, and then bringing the bottom of the test zone in contact with a piece of blotting paper. Once dry, the test zones were scanned using a fluorescent scanner (Typhoon Trio, General Electric Co. set to 526-nm excitation, 532-nm emission). The fluorescence intensity of each test zone was quantified by analyzing the image using ImageQuant.

Blocking the Paper. Test zones were blocked with a blocking buffer $(1 \mu \mathrm{L}$ of $0.05 \%(\mathrm{w} / \mathrm{v})$ Tween-20 and 1\% (w/v) BSA in PBS) and allowed to dry for 10 minutes. Solutions of $50-\mathrm{ng} / \mu \mathrm{L}, 25-\mathrm{ng} / \mu \mathrm{L}, 12.5-\mathrm{ng} / \mu \mathrm{L}$, and $0-\mathrm{ng} / \mu \mathrm{LTRITC}-\mathrm{labeled}$ rabbit IgG in 
PBS $(3 \mu \mathrm{L})$ were spotted in test zones that were either blocked or not blocked. After incubating for one minute, the test zones were washed with PBS $(2 \times 10 \mu \mathrm{L})$ as described above. Once dry the test zones were scanned and the fluorescence intensity of each zone was measured.

Removing Unbound Proteins from Paper by Washing. Solutions of 50-ng/ $\mu \mathrm{L}$ and 0 -ng/ $\mu$ LTRITC-labeled rabbit IgG in PBS $(3 \mu \mathrm{L})$ were spotted in blocked test zones, incubated for one minute and then washed using nine different washing protocols. The washing protocols tested the number of washes (from 1 to 3), and the volume of PBS used for each was $(5,10$ and $15 \mu \mathrm{L})$. Once dry, the test zones were scanned and the fluorescence intensity of each zone was measured.

Indirect P-ELISA for Rabbit IgG Detection. Rabbit IgG $(3 \mu \mathrm{L})$ in ten fold dilutions ( $67 \mathrm{mM}$ to $670 \mathrm{nM}$, corresponding to 67 pmoles to 670 amoles of IgG per zone spotted on the paper) was immobilized in test zones; PBS was used as a negative control. The test zones were blocked, and a solution of ALP-conjugated anti-rabbit IgG produced in goat (3 $\mu \mathrm{L}$ of a 1:1000 dilution the of stock antibody solution in $0.05 \%$ Tween-20 in PBS) was added to the test zones and allowed to incubate for one minute. The test zones were then washed with PBS $(2 \times 10 \mu \mathrm{L})$, and the ALP substrate solution ( $3 \mu \mathrm{L}$ of $2.68 \mathrm{mM}$ BCIP, $1.8 \mathrm{mM} \mathrm{NBT}, 5 \mathrm{mM} \mathrm{MgCl} 2,100 \mathrm{mM} \mathrm{NaCl}, 0.05 \%$ Tween in $100 \mathrm{mM}$ Tris buffer, $\mathrm{pH}$ 9.5) was added to each well. After 30 minutes, the test zones were scanned (Perfection 1640SU scanner, EPSON Corp., set to color photo scanning, 600 dpi 
resolution), and the image was analyzed in grayscale using ImageJ. All the antibodies and reagents were purchased from Sigma Aldrich.

P-ELISA for HIV-1 Envelope Antigens Detection. HIV-1 envelope antigen (gp41, $1 \mathrm{mg} / \mathrm{mL}$ ) was diluted 1/10 in PBS and immobilized in test zones. The test zones were blocked using 1\% (w/v) BSAand serum from HIV-1 infected patients and healthy control patients was added to the test zones, incubated and washed. A solution of ALPconjugated anti-human HIV-1 produced in goat ( $3 \mu \mathrm{L}$ of a 1:1000 dilution the of stock antibody solution in $0.05 \%$ Tween-20 in PBS) was then incubated in the test zones and washed. The procedure is the same as that we developed for rabbit IgG. Five microliters of the ALP substrate solution was added to each well; after 30 minutes, the results were scanned and analyzed in ImageJ. The HIV-1 envelope (gp41) antigen was purchased from ViroGen (Cambridge, MA). The serum samples were purchased from Golden West Biologicals (Temecula, CA). 